\title{
THE KAPLANSKY RADICAL OF A QUADRATIC FIELD EXTENSION
}

\author{
KARIM JOHANNES BECHER AND DAVID B. LEEP
}

\begin{abstract}
The radical of a field consists of all nonzero elements that are represented by every binary quadratic form representing 1 . Here, the radical is studied in relation to local-global principles, and further in its behaviour under quadratic field extensions. In particular, an example of a quadratic field extension is constructed where the natural analogue to the square-class exact sequence for the radical fails to be exact. This disproves a conjecture of Kijima and Nishi.

Keywords: quadratic form, local-global principle, quasi-pythagorean field, function field, power series field, quadratic field extension
\end{abstract}

Classification (MSC 2010): 11E04, 11E81, 12D15, 12F05, 13J05, 14H05

\section{INTRODUCTION}

Let $K$ be a field of characteristic different from 2. Let $K^{\times}$denote the multiplicative group of $K, \sum K^{\times 2}$ the subgroup of nonzero sums of squares in $K$, and $D_{K}\langle 1, a\rangle$ the subgroup of $K^{\times}$consisting of the nonzero elements represented by the binary quadratic form $X^{2}+a Y^{2}$, for any $a \in K^{\times}$. The object of study in this article is the subgroup

$$
\mathrm{R}(K)=\bigcap_{a \in K^{\times}} D_{K}\langle 1, a\rangle
$$

of $K^{\times}$, called the (Kaplansky) radical of $K$. This object was first studied by I. Kaplansky for fields over which there exists a unique quaternion division algebra [7]. It was investigated in more generality by C.M. Cordes [4], who baptized it the Kaplansky radical and observed that in several statements about quadratic forms over $K$ one can replace $K^{\times 2}$ by $\mathrm{R}(K)$. We refer to [11, Chap. XII, Sect. $6 \& 7$ ] for an introduction to the Kaplansky radical. By [11, Chap.XII, (6.1)] the radical is further characterized as $\mathrm{R}(K)=\left\{c \in K^{\times} \mid D_{K}\langle 1,-c\rangle=K^{\times}\right\}$.

In this article we continue the study of the radical. In Section 2 we consider the position of the radical within the inclusions $K^{\times 2} \subseteq \mathrm{R}(K) \subseteq \sum K^{\times 2}$. In Section 3 we study fields satisfying a local-global principle for quadratic forms and derive a determination of the radical as the set of elements that are locally squares. In Section 4 we revisit the behavior of the radical under quadratic field extensions and disprove a conjecture by D. Kijima and M. Nishi discussed in [8], [9], and [6].

Date: 16 July, 2013. 


\section{Position of the RAdical}

We have the inclusions $K^{\times 2} \subseteq \mathrm{R}(K) \subseteq D_{K}\langle 1,1\rangle \subseteq \sum K^{\times 2}$. We first consider the two extremal cases for the position of the radical with respect to these inclusions. We say that $K$ is radical-free if $\mathrm{R}(K)=K^{\times 2}$.

2.1. Proposition. Assume that $\left|K^{\times} / K^{\times 2}\right| \geq 4$ and there exists $t \in K^{\times}$such that $D_{K}\langle 1, t\rangle=K^{\times 2} \cup t K^{\times 2}$ and $D_{K}\langle 1,-t\rangle=K^{\times 2} \cup-t K^{\times 2}$. Then $K$ is radical-free.

Proof. We may choose an element $a \in K^{\times} \backslash\left(K^{\times 2} \cup t K^{\times 2}\right)$. Then $a \notin D_{K}\langle 1, t\rangle$ and thus $-t \notin D_{K}\langle 1,-a\rangle$, whereby $\mathrm{R}(K) \subseteq D_{K}\langle 1,-t\rangle \cap D_{K}\langle 1,-a\rangle=K^{\times 2}$.

By a $\mathbb{Z}$-valuation we mean a valuation with value group $\mathbb{Z}$. For a $\mathbb{Z}$-valuation $v$ on $K$ we denote by $K_{v}$ the corresponding completion.

2.2. Corollary. Assume that $K$ is henselian with respect to a $\mathbb{Z}$-valuation whose residue field is of characteristic different from 2 and not quadratically closed. Then $K$ is radical-free.

Proof. It follows from the hypotheses that $\left|K^{\times} / K^{\times 2}\right| \geq 4$. Moreover, any $t \in$ $K^{\times}$that has odd value with respect to the given valuation will be such that $D_{K}\langle 1, t\rangle=K^{\times 2} \cup t K^{\times 2}$ and $D_{K}\langle 1,-t\rangle=K^{\times 2} \cup-t K^{\times 2}$. Hence, the statement follows from (2.1).

By [11, Chap. XII, Sect. 6], if $K$ is a finite extension of the field of $p$-adic numbers $\mathbb{Q}_{p}$ for a prime number $p$, then $K$ is radical-free; for $p \neq 2$ this can be seen from $(2.2)$.

2.3. Proposition. The following are equivalent:

(i) $\mathrm{R}(K)=\sum K^{\times 2}$;

(ii) $\mathrm{R}(K)=D_{K}\langle 1,1\rangle$;

(iii) $I_{t}^{2} K=0$;

(iv) every torsion 2-fold Pfister form over $K$ is hyperbolic.

Proof. This follows from [11, Chap. XI, (4.1) and (4.5)] for $n=2$.

Condition $(i v)$ corresponds to Property $\left(A_{2}\right)$ in the terminology of [5], treated also in [11, Chap. XI, Sect. 4]. Following [9] we say that the field $K$ is quasipythagorean if it satisfies the equivalent conditions in (2.3). By [11, [Chap. XI, (6.26)] this is further equivalent to having that the $u$-invariant of $K$ is at most 2 . For example, by [11, Chap. XI, (4.10)], any extension of transcendence degree one of a real closed field is quasi-pythagorean.

In [4] Cordes gave an example of a field $K$ with $K^{\times 2} \subsetneq \mathrm{R}(K) \subsetneq \sum K^{\times 2}$ and asked whether one can have such examples where $K^{\times} / K^{\times 2}$ is finite. M. Kula [10] and L. Berman [2] independently constructed such examples. We give another example where $K$ is a nonreal algebraic extension of $\mathbb{Q}$ having 8 square classes. 
2.4. Example. The integers $-2,-5$ and 7 are squares in $\mathbb{Q}_{3}$. Hence, $\mathbb{Q}_{3}$ contains the field $\mathbb{Q}(\sqrt{-2}, \sqrt{-5})$. Moreover, 7 is not a square in $\mathbb{Q}(\sqrt{-2}, \sqrt{-5})$. Consider the set of subfields of $\mathbb{Q}_{3}$ that are algebraic extensions of $\mathbb{Q}(\sqrt{-2}, \sqrt{-5})$ and in which 7 is not a square. By Zorn's Lemma, we may choose a maximal element $K$ in this set. Then $K$ is a field whose unique quadratic extension contained in $\mathbb{Q}_{3}$ is $K(\sqrt{7})$. As the four square classes of $\mathbb{Q}_{3}$ are represented by $1,2,3$ and 6 , it follows that the classes of $2,3,7$ form an $\mathbb{F}_{2}$-basis of the square class group $K^{\times} / K^{\times 2}$. In particular $\left|K^{\times} / K^{\times 2}\right|=8$.

As $\mathbb{Q}_{3}^{\times}=K^{\times} \mathbb{Q}_{3}^{\times 2}$ we conclude that $\mathrm{R}(K) \subseteq \mathrm{R}\left(\mathbb{Q}_{3}\right)$. As $\mathbb{Q}_{3}$ is radical-free, we obtain that $\mathrm{R}(K) \subseteq K^{\times} \cap \mathbb{Q}_{3}^{\times 2}=K^{\times 2} \cup 7 K^{\times 2}$. Since $2=3^{2}-7,3=$ $(\sqrt{-2} \cdot \sqrt{-5})^{2}-7$ and $2 \cdot 3 \cdot 7=7^{2}-7$, we see that $D_{K}\langle 1,-7\rangle=K^{\times}$. This shows that $\mathrm{R}(K)=K^{\times 2} \cup 7 K^{\times 2}$.

The number of square classes in (2.4) is minimal for having a nontrivial radical, by the following statement.

2.5. Proposition. If $K^{\times 2} \subsetneq \mathrm{R}(K) \subsetneq \sum K^{\times 2}$ then $\left|K^{\times} / K^{\times 2}\right| \geq 8$.

Proof. By [11, Chap. XII, (6.10)], if $\mathrm{R}(K)$ has index two in $K^{\times}$, then $K$ is real and thus $\mathrm{R}(K)=\sum K^{\times 2}$. Hence, if $\mathrm{R}(K) \subsetneq \sum K^{\times 2}$ then $\left|K^{\times} / \mathrm{R}(K)\right| \geq 4$.

\section{THE RADICAL AS THE GROUP OF LOCAL SQUARES}

In certain fields satisfying a local-global principle for isotropy of quadratic forms, the radical consists of the elements that are squares locally.

3.1. Proposition. Let $\left(K_{\wp}\right)_{\wp \in \mathcal{P}}$ be a family of extension fields of $K$ such that $K_{\wp}^{\times}=K^{\times} K_{\wp}^{\times 2}$ for every $\wp \in \mathcal{P}$. Then

$$
\mathrm{R}(K) \subseteq \bigcap_{\wp \in \mathcal{P}}\left(K^{\times} \cap \mathrm{R}\left(K_{\wp}\right)\right) .
$$

This inclusion is an equality if every 3-dimensional anisotropic quadratic form $\varphi$ over $K$ stays anisotropic over $K_{\wp}$ for some $\wp \in \mathcal{P}$.

Proof. For $c \in \mathrm{R}(K)$ and $\wp \in \mathcal{P}$, one has $K_{\wp}^{\times}=K^{\times} K_{\wp}^{\times 2}=D_{K}\langle 1,-c\rangle K_{\wp}^{\times 2}$ and thus $c \in \mathrm{R}\left(K_{\wp}\right)$. This shows that $\mathrm{R}(K) \subseteq \bigcap_{\wp \in \mathcal{P}}\left(K^{\times} \cap \mathrm{R}\left(K_{\wp}\right)\right)$.

Consider now $c \in K^{\times} \backslash \mathrm{R}(K)$. As $D_{K}\langle 1,-c\rangle \subsetneq K^{\times}$there exists $b \in K^{\times}$such that the form $\langle 1,-c,-b\rangle$ over $K$ is anisotropic. If $\wp \in \mathcal{P}$ is such that $\langle 1,-c,-b\rangle$ stays anisotropic over $K_{\wp}$, then we conclude that $c \notin \mathrm{R}\left(K_{\wp}\right)$. Therefore, if every 3 -dimensional anisotropic quadratic form $\varphi$ over $K$ stays anisotropic over $K_{\wp}$ for some $\wp \in \mathcal{P}$, we obtain that $\mathrm{R}(K)=\bigcap_{\wp \in \mathcal{P}}\left(K^{\times} \cap \mathrm{R}\left(K_{\wp}\right)\right)$.

3.2. Proposition. Let $\Omega$ be a set of $\mathbb{Z}$-valuations of $K$ whose residue fields are of characteristic different from 2 and not quadratically closed. The following hold:

(a) One has $\mathrm{R}(K) \subseteq \bigcap_{v \in \Omega}\left(K^{\times} \cap K_{v}^{\times 2}\right)$.

(b) If $\bigcap_{v \in \Omega}\left(K^{\times} \cap K_{v}^{\times 2}\right)=K^{\times 2}$, then $K$ is radical-free. 
(c) If for every 3-dimensional anisotropic quadratic form $\varphi$ over $K$ there exists $v \in \Omega$ such that $\varphi$ stays anisotropic over $K_{v}$, then $\mathrm{R}(K)=\bigcap_{v \in \Omega}\left(K^{\times} \cap K_{v}^{\times 2}\right)$.

Proof. For $v \in \Omega$, we have $K_{v}^{\times}=K^{\times} K_{v}^{\times 2}$ as well as $\mathrm{R}\left(K_{v}\right)=K_{v}^{\times 2}$ by (2.2). Therefore (3.1) applies and yields $(a)$ and $(c)$. Moreover $(a)$ implies $(b)$.

Using (3.2) we retrieve the well-known fact that any number field is radical-free:

3.3. Example. Let $K$ be a global field of characteristic different from 2 and let $\Omega$ denote the set of all non-dyadic $\mathbb{Z}$-valuations of $K$. As $K$ has only finitely many archimedean and non-archimedean dyadic places, the Global-Square-Theorem (cf. $[13,(65: 15)])$ implies that $\bigcap_{v \in \Omega}\left(K^{\times} \cap K_{v}^{\times 2}\right)=K^{\times 2}$. Hence, (3.2) yields that $K$ is radical-free.

3.4. Proposition. Assume that $K$ is a rational function field in one variable over a field $k$. Let $\Omega$ denote the set of $\mathbb{Z}$-valuations on $K$ that are trivial on $k$. Then

$$
\bigcap_{v \in \Omega}\left(K^{\times} \cap K_{v}^{\times 2}\right)=K^{\times 2}
$$

Moreover, if $k(\sqrt{-1})$ is not quadratically closed then $K$ is radical-free.

Proof. Let $T \in K$ be such that $K=k(T)$. Any square class of $K$ is given by a square-free polynomial $f \in k[T]$. Note that $v(f)$ is 0 or 1 for every $v \in \Omega$ corresponding to an irreducible monic polynomial in $k[T]$. If $v(f)=1$ for one such $v$, then $f \notin K_{v}^{\times 2}$. If $v(f)=0$ for all such $v$, then $f \in k$. Finally, if $f \in k^{\times} \backslash k^{\times 2}$, then $f \notin K_{v}^{\times 2}$ where $v$ is the valuation given by $T$. This together yields the claimed equality.

Assume now that $k(\sqrt{-1})$ is not quadratically closed. It follows that no finite extension of $k$ is quadratically closed. In fact, if there were a finite field extension $k^{\prime} / k$ such that $k^{\prime}$ is quadratically closed, then $k^{\prime}$ would contain $k(\sqrt{-1})$ and [11, Chap. VIII, (5.11)] would imply that $k(\sqrt{-1})$ is quadratically closed. In particular, for $v \in \Omega$, the residue field of $v$ is not quadratically closed. Thus $K$ is radical-free, by (3.2).

3.5. Corollary. Assume that $K$ contains a subfield $k$ such that $K / k$ is purely transcendental of transcendence degree at least two. Then $K$ is radical-free.

Proof. Let $\mathcal{X}$ be a transcendence basis of $K / k$ with $K=k(\mathcal{X})$. Choose $x \in \mathcal{X}$ and put $\mathcal{X}^{\prime}=\mathcal{X} \backslash\{x\}$ and $K_{0}=k\left(\mathcal{X}^{\prime}\right)$. Then $K=K_{0}(x)$. As $\mathcal{X}^{\prime} \neq \emptyset$ by the hypothesis, we have that $K_{0}(\sqrt{-1})=k(\sqrt{-1})\left(\mathcal{X}^{\prime}\right)$ is not quadratically closed. Hence, we conclude from (3.4) that $\mathrm{R}(K)=K^{\times 2}$.

3.6. Question. Assume that $K$ is a finitely generated field extension of transcendence degree at least two of another field $k$. Is then $K$ radical-free? Is every non-square in $K$ a non-square in the completion of a $\mathbb{Z}$-valuation on $K$ that is trivial on $k$ and whose residue-field is an algebraic function field over $k$ ? 
3.7. Theorem. Assume that $K=k\left(\left(X_{1}, \ldots, X_{n}\right)\right)$ for a field $k$ of characteristic different from 2. Let $\Omega$ denote the set of $\mathbb{Z}$-valuations on $K$ corresponding to the localizations of $k \llbracket X_{1}, \ldots, X_{n} \rrbracket$ at its height one prime ideals. Then

$$
\bigcap_{v \in \Omega}\left(K^{\times} \cap K_{v}^{\times 2}\right)=K^{\times 2}
$$

In particular, $K$ is radical-free unless $k$ is quadratically closed and $n=1$.

Proof. Let $A=k \llbracket X_{1}, \ldots, X_{n} \rrbracket$. Note that $A$ is a unique factorization domain by $[12,(20.3)$ and $(20.8)]$, and noetherian by $[1,(10.27)]$. In particular, by $[12$, (20.1)] any height one prime ideal in $A$ is principal.

Consider an arbitrary element $a \in K^{\times}$. We may write $a=u \cdot p_{1} \ldots p_{r} \cdot x^{2}$ where $u \in A^{\times}, x \in K^{\times}, r \geq 0$, and where $p_{1}, \ldots, p_{r}$ are pairwise non-associate prime elements of $A$. Let $c$ denote the constant term of $u$ as a power series. Then $c^{-1} u$ is a 1 -unit in $A$, and therefore a square in $A$. Note that, for $v \in \Omega$, we have that $v(a)$ is odd if $v$ is associated to one of the prime elements $p_{1}, \ldots, p_{r}$, and $v(a)$ is even otherwise. Assume now that $a \in \bigcap_{v \in \Omega}\left(K^{\times} \cap K_{v}^{\times 2}\right)$. Then $v(a)$ is even for every $v \in \Omega$, whereby $r=0, a=u x^{2}$, and $a K^{\times 2}=c K^{\times 2}$. Let $w$ be the $\mathbb{Z}$-valuation associated to the irreducible element $X_{n}$ in $A$. Note that $K_{w}=k\left(\left(X_{1}, \ldots, X_{n-1}\right)\right)\left(\left(X_{n}\right)\right)$. It follows that $c \in k^{\times} \cap K_{w}^{\times 2}=k^{\times 2}$, whereby $a \in K^{\times 2}$. This argument shows that $\bigcap_{v \in \Omega}\left(K^{\times} \cap K_{v}^{\times 2}\right)=K^{\times 2}$.

Furthermore, if $n=1$, then $K=k\left(\left(X_{1}\right)\right)$ and it follows by $(2.2)$ that $K$ is radical-free unless $k$ is quadratically closed. Assume now that $n \geq 2$. The residue field of any valuation $v \in \Omega$ is $k$-isomorphic to a finite extension of $k\left(\left(X_{1}, \ldots, X_{n-1}\right)\right)$ and therefore is not quadratically closed. Using (3.2) the proven equality yields that $K$ is radical-free.

\section{The RAdical COMPleX FOR a QUADRATIC EXTENSION}

We consider a finite field extension $L / K$ and ask about the relations between $\mathrm{R}(K)$ and $\mathrm{R}(L)$. After a first general result, we shall focus on the case of a quadratic extension. Let $N_{L / K}: L^{\times} \longrightarrow K^{\times}$be the group homomorphism given by the norm map.

4.1. Proposition. We have $N_{L / K}(\mathrm{R}(L)) \subseteq \mathrm{R}(K)$.

Proof. For $a \in K^{\times}$, as $\mathrm{R}(L) \subseteq D_{L}\langle 1, a\rangle$ we have that $N_{L / K}(\mathrm{R}(L)) \subseteq D_{K}\langle 1, a\rangle$, by [11, Chap. VII, (4.3)]. Hence, $N_{L / K}(\mathrm{R}(L)) \subseteq \bigcap_{a \in K^{\times}} D_{K}\langle 1, a\rangle$.

For the remainder of this section we consider the case where $L / K$ is a quadratic field extension. We denote by $\iota_{L / K}$ the inclusion homomorphism $K^{\times} \longrightarrow L^{\times}$.

4.2. Proposition. Assume that $L \simeq K(\sqrt{a})$ where $a \in K^{\times}$. For any $b \in K^{\times}$we have that

$$
D_{L}\langle 1,-b\rangle \cap K^{\times}=D_{K}\langle 1,-b\rangle \cdot D_{K}\langle 1,-a b\rangle .
$$

Proof. See e.g. [3, (2.4)]. 
The following was shown in [4, Cor. of Prop. 3; Prop. 5] as a partial analogue to the square-class exact sequence in [11, Chap. VII, (3.8)].

4.3. Proposition. We have $\mathrm{R}(K) \subseteq \mathrm{R}(L)$ and $N_{L / K}\left(K^{\times} \mathrm{R}(L)\right) \subseteq \mathrm{R}(K)$. In particular, the maps $\iota_{L / K}$ and $N_{L / K}$ induce a complex

$$
K^{\times} / \mathrm{R}(K) \longrightarrow L^{\times} / \mathrm{R}(L) \longrightarrow K^{\times} / \mathrm{R}(K),
$$

which is exact if and only if $K^{\times} \mathrm{R}(L)=N_{L / K}^{-1}(\mathrm{R}(K))$.

Proof. Consider $b \in K^{\times}$. By the Norm Principle [11, Chap. VII (5.10)] we have that

$$
N_{L / K}^{-1}\left(D_{K}\langle 1,-b\rangle\right)=K^{\times} D_{L}\langle 1,-b\rangle .
$$

Hence, if $D_{K}\langle 1,-b\rangle=K^{\times}$, then $D_{L}\langle 1,-b\rangle=L^{\times}$. This shows that $\mathrm{R}(K) \subseteq \mathrm{R}(L)$.

Since $N_{L / K}(\mathrm{R}(L)) \subseteq \mathrm{R}(K)$ by $(4.1)$ and $N_{L / K}\left(K^{\times}\right) \subseteq K^{\times 2}$, it follows that $N_{L / K}\left(K^{\times} \mathrm{R}(L)\right) \subseteq \mathrm{R}(K)$. The statement follows from this.

There are examples of quadratic field extensions $L / K$ where $K$ is radicalfree whereas $L$ is not. For example, in [2, Section 2], for any positive integer $n$ a real pythagorean field $K$ is constructed such that $L=K(\sqrt{-1})$ satisfies $\left|L^{\times} / \mathrm{R}(L)\right|=4$ and $\left|\mathrm{R}(L) / L^{\times 2}\right|=2^{n}$.

D. Kijima and M. Nishi [8] raised the question whether the complex in (4.3) is always exact. We will show that the answer is negative by providing a construction that produces counter-examples. To simplify the discussion of the problem, we say that the quadratic field extension $L / K$ is radical-exact if the equality $K^{\times} \mathrm{R}(L)=N_{L / K}^{-1}(\mathrm{R}(K))$ holds, that is, if the complex in (4.3) is exact.

4.4. Corollary. Let $L / K$ be a quadratic field extension such that $N_{L / K}$ is surjective. Then $\mathrm{R}(K)=K^{\times} \cap \mathrm{R}(L)$ and the maps $\iota_{L / K}$ and $N_{L / K}$ induce a complex

$$
1 \longrightarrow K^{\times} / \mathrm{R}(K) \longrightarrow L^{\times} / \mathrm{R}(L) \longrightarrow K^{\times} / \mathrm{R}(K) \longrightarrow 1,
$$

which is exact on the left and on the right. In particular, this is an exact sequence provided that $L / K$ is radical-exact.

Proof. Let $a \in K^{\times}$be such that $L=K(\sqrt{a})$. As $N_{L / K}$ is surjective, the norm form $\langle 1,-a\rangle$ of $L / K$ is universal over $K$, whereby $a \in \mathrm{R}(K)$. This further shows that the complex is exact on the right.

Consider an arbitrary element $b \in K^{\times}$. As $a \in \mathrm{R}(K)$, by [11, Chap. XII, (6.3)] we have that $D_{K}\langle 1,-b\rangle=D_{K}\langle 1,-a b\rangle$. Using (4.2) we thus obtain that $D_{K}\langle 1,-b\rangle=K^{\times} \cap D_{L}\langle 1,-b\rangle$. Therefore, if the form $\langle 1,-b\rangle$ is universal over $L$, it is also universal over $K$. This shows that $K^{\times} \cap \mathrm{R}(L) \subseteq \mathrm{R}(K)$. Since by (4.3) the opposite inclusion also holds, we obtain that $\mathrm{R}(K)=K^{\times} \cap \mathrm{R}(L)$. In particular, the complex is exact on the left.

The rest follows from (4.3).

The following recovers $[9,(2.13)]$. 
4.5. Proposition. Assume that $L \simeq K(\sqrt{d})$ with $d \in \sum K^{\times 2}$. Then $K$ is quasipythagorean if and only if $L$ quasi-pythagorean, and in this case $L / K$ is radicalexact.

Proof. This claimed equivalence is $[5,(4.10) ;(4.5)]$ for $n=2$. Assume now that $K$ and $L$ are quasi-pythagorean. Using the Norm Principle [11, Chap. VII, (5.10)], we obtain that

$$
N_{L / K}^{-1}(\mathrm{R}(K))=N_{L / K}^{-1}\left(D_{K}\langle 1,1\rangle\right)=K^{\times} D_{L}\langle 1,1\rangle=K^{\times} \mathrm{R}(L),
$$

showing that $L / K$ is radical-exact.

4.6. Proposition. Let $L / K$ be a quadratic field extension with $\mathrm{R}(L)=L^{\times 2}$. Then $\mathrm{R}(K) \subseteq K^{\times} \cap L^{\times 2}$ and $K^{\times} \mathrm{R}(L) \subseteq N_{L / K}^{-1}(\mathrm{R}(K))$, and exactly one of the two inclusions is strict.

Proof. Since $\mathrm{R}(L)=L^{\times 2}$ we have that

$$
K^{\times} \mathrm{R}(L)=K^{\times} L^{\times 2}=N_{L / K}^{-1}\left(K^{\times 2}\right) .
$$

By (4.3) we obtain that $\mathrm{R}(K) \subseteq K^{\times} \cap \mathrm{R}(L)=K^{\times} \cap L^{\times 2}$. Let $a \in K^{\times}$be such that $L=K(\sqrt{a})$. Then $K^{\times} \cap L^{\times 2}=K^{\times 2} \cup a K^{\times 2}$. Hence, either $\mathrm{R}(K)=K^{\times 2}$ or $\mathrm{R}(K)=K^{\times 2} \cup a K^{\times 2}$.

If $\mathrm{R}(K)=K^{\times 2}$, then $\mathrm{R}(K) \subsetneq K^{\times} \cap L^{\times 2}$ and from the above we obtain that $K^{\times} \mathrm{R}(L)=N_{L / K}^{-1}(\mathrm{R}(K))$. Assume now that $\mathrm{R}(K)=K^{\times 2} \cup a K^{\times 2}$. Then in particular $a \in D_{K}\langle 1,-a\rangle=N_{L / K}\left(L^{\times}\right)$. Hence, we obtain that

$$
K^{\times} \mathrm{R}(L)=N_{L / K}^{-1}\left(K^{\times 2}\right) \subsetneq N_{L / K}^{-1}\left(K^{\times 2} \cup a K^{\times 2}\right)=N_{L / K}^{-1}(\mathrm{R}(K)) .
$$

4.7. Lemma. Let $a \in K^{\times} \backslash K^{\times 2}$. Let $\mathcal{C}$ be the set of isomorphism classes of smooth conics over $K$ having a $K(\sqrt{a})$-rational point. For $C \in \mathcal{C}$ let $K(C)$ denote the corresponding function field, determined by $C$ up to $K$-isomorphism. Let $K^{\prime}$ be a field composite of all $K(C)$ with $C \in \mathcal{C}$. Then $K^{\times} \subseteq D_{K^{\prime}}\langle 1,-a\rangle$ and the extension $K^{\prime}(\sqrt{a}) / K(\sqrt{a})$ is purely transcendental.

Proof. The field $K^{\prime}(\sqrt{a})$ is the compositum of the function fields $K(\sqrt{a})(C)$ for all $C \in \mathcal{C}$. Since every $C \in \mathcal{C}$ is rational over $K(\sqrt{a})$, the field $K^{\prime}(\sqrt{a})$ is a compositum of rational function fields in one variable over $K(\sqrt{a})$, thus a purely transcendental extension of $K(\sqrt{a})$.

By construction, every smooth conic over $K$ that has a $K(\sqrt{a})$-rational point has a $K^{\prime}$-rational point. Hence, for any $b \in K^{\times}$the ternary quadratic form $\langle 1,-a,-b\rangle$ becomes isotropic over $K^{\prime}$. Thus $K^{\times} \subseteq D_{K^{\prime}}\langle 1,-a\rangle$.

4.8. Theorem. Let $L / K$ be a quadratic field extension. There exists a field extension $K^{\prime} / K$ that is linearly disjoint to $L / K$ and such that $L K^{\prime}$ is radicalfree, $\mathrm{R}\left(K^{\prime}\right)=K^{\prime \times} \cap\left(L K^{\prime}\right)^{\times 2}$, and $L K^{\prime} / K^{\prime}$ not radical-exact. 
Proof. Let $L=K(\sqrt{a})$ with $a \in K^{\times}$. We define a tower of extension fields $\left(K_{i}\right)_{i \in \mathbb{N}}$ of $K$ by letting $K_{0}=K$ and, $K_{i+1}$ the field composite over $K_{i}$ of all $K_{i}(C)$ where $C$ runs over the isomorphism classes of conics over $K_{i}$ having a $K_{i}(\sqrt{a})$-rational point. Let $K^{\prime}$ denote the direct limit of the tower of fields $\left(K_{i}\right)_{i \in \mathbb{N}}$. For $i \in \mathbb{N}$, then $K^{\prime} / K_{i}$ is linearly disjoint to any algebraic extension of $K_{i}$ and, moreover, by (4.7) $K_{i+1}(\sqrt{a}) / K_{i}(\sqrt{a})$ is purely transcendental and every element of $K_{i}^{\times}$is represented over $K_{i+1}$ by the form $\langle 1,-a\rangle$. It follows that $K^{\prime} / K$ is linearly disjoint to $L / K$, that the form $\langle 1,-a\rangle$ is universal over $K^{\prime}$, and that $K^{\prime} L=K^{\prime}(\sqrt{a})$ is a purely transcendental extension of $K(\sqrt{a})$, whereby $K^{\prime} L$ is radical-free by (3.5). Note that $K^{\prime \times} \cap\left(K^{\prime} L\right)^{\times 2}=K^{\prime \times 2} \cup a K^{\prime \times 2} \subseteq \mathrm{R}\left(K^{\prime}\right)$. Using (4.6) we conclude that $\mathrm{R}\left(K^{\prime}\right)=K^{\prime \times} \cap\left(K^{\prime} L\right)^{\times 2}$ and that $K^{\prime} L / K^{\prime}$ is not radical-exact.

\section{REFERENCES}

[1] M.F. Atiyah, I.G. Macdonald. Introduction to commutative algebra. Addison-Wesley Publishing Co., Reading, Mass.-London-Don Mills, Ont., 1969.

[2] L. Berman. Pythagorean fields and the Kaplansky radical. J. Algebra 61 (1979): 497-507.

[3] K.J. Becher, J. Van Geel. Sums of squares in function fields of hyperelliptic curves. Math. Z. 261 (2009): 829-844.

[4] C.M. Cordes. Kaplanskys radical and quadratic forms over nonreal fields. Acta Arith. 28 (1975): 253-261.

[5] R. Elman, T. Y. Lam. Quadratic forms under algebraic extensions. Math. Ann. 219 (1976): 21-42.

[6] T. Iwakami, D. Kijima, M. Nishi. Kaplansky's radical and Hilbert theorem 90. III. Hiroshima Math. J. 15 (1985): 81-88.

[7] I. Kaplansky. Fröhlich's local quadratic forms. J. Reine Angew. Math. 239-240 (1969): 74-77.

[8] D. Kijima, M. Nishi. Kaplansky's radical and Hilbert theorem 90. Hiroshima Math. J. 11 (1981): 443-456.

[9] D. Kijima, M. Nishi. Kaplansky's radical and Hilbert theorem 90. II. Hiroshima Math. J. 13 (1983): 29-37.

[10] M. Kula. Fields with non-trivial Kaplansky's radical and finite square class number. Acta Arith. 38 (1980/1981): 411-418.

[11] T.Y. Lam. Introduction to quadratic forms over fields. Graduate Studies in Mathematics, 67, American Mathematical Society, Providence, RI, 2005.

[12] H. Matsumura. Commutative ring theory. Cambridge Studies in Advanced Mathematics Vol. 8, Cambridge University Press, Cambridge, 1986.

[13] O.T. O'Meara. Introduction to quadratic forms. Reprint of the 1973 edition (Grundlehren der Mathematischen Wissenschaften, 117). Classics in Mathematics. Springer-Verlag, Berlin, 2000.

Departement Wiskunde-Informatica, Universiteit Antwerpen, Belgium /

Zukunftskolleg, Universität Konstanz, Germany.

E-mail address: becher@maths.ucd.ie

Department of Mathematics, University of Kentucky, Lexington, KY 40506 0027, USA.

E-mail address: leep@email.uky.edu 